\title{
Erythropeltidaceen (Bangiophyceae, Rhodophyta) von Helgoland
}

\author{
P. Kornmann \& P.-H. Sahling \\ Biologische Anstalt Helgoland (Meeresstation); D-2192 Helgoland, \\ Bundesrepublik Deutschland
}

\begin{abstract}
Erythropeltidaceae (Bangiophyceae, Rhodophyta) from Helgoland. Ontogenesis and reproduction of the Helgolandian taxa of the Erythropeltidaceae have been studied. In all species monospores are only produced from differentiated sporangia. Filamentous Conchocelis-like stages have not been observed. Sexual reproduction was formerly demonstrated in the heteromorphous genus Erythrotrichopeltis (Kornmann, 1984). Based on these features a revised classification for the family is presented. Porphyropsis imperfecta, a new species, is a widespread epiphyte in sublittoral habitats.
\end{abstract}

\section{EINLEITUNG}

Zwei Arbeiten neueren Datums führen in die Literatur über die Erythropeltidaceen ein. Garbary et al. (1980) gliedern die Bangiophyceen neu und führen die Familie zusammen mit den Boldiaceen und Compsopogonaceen einer eigenen Ordnung, Erythropeltidales, zu. Kennzeichnendes Merkmal der Ordnung ist die Entstehungsweise des Monosporangiums aus einer durch eine schräge Wand geteilten undifferenzierten Zelle.

Dixon \& Murray (1981) fassen die wenigen und nur schwer zu interpretierenden Angaben über die Fortpflanzung und Entwicklung der Erythropeltidaceen zusammen: "Little is known of life histories in members of this family" (Dixon \& Murray, 1981; p. 93). Durch die entwicklungsgeschichtliche Untersuchung der bei Helgoland vorkommenden Gattungen wird diese Lücke weitgehend geschlossen. Die fünf untersuchten Gattungen sind morphologisch und entwicklungsgeschichtlich klar gekennzeichnet. Alle vermehren sich durch Monosporen; in der heteromorphen Gattung Erythrotrichopeltis erzeugt die fädige Phase zusätzlich Geschlechtszellen (Kornmann, 1984). Entwicklungsstadien, die als Conchocelis gedeutet werden könnten, wurden nicht beobachtet. Die folgende Gliederung der Familie enthält die wesentlichen Ergebnisse der entwicklungsgeschichtlichen Untersuchung.

A. Lebenszyklus isomorph

I. Thallus fädig, ohne primäre Haftscheibe

Erythrotrichia

II. Thallus scheibenförmig

1. Die Scheibe schließt sich aus akropetal verzweigten Fäden zusammen

2. Scheiben mit Randwachstum 

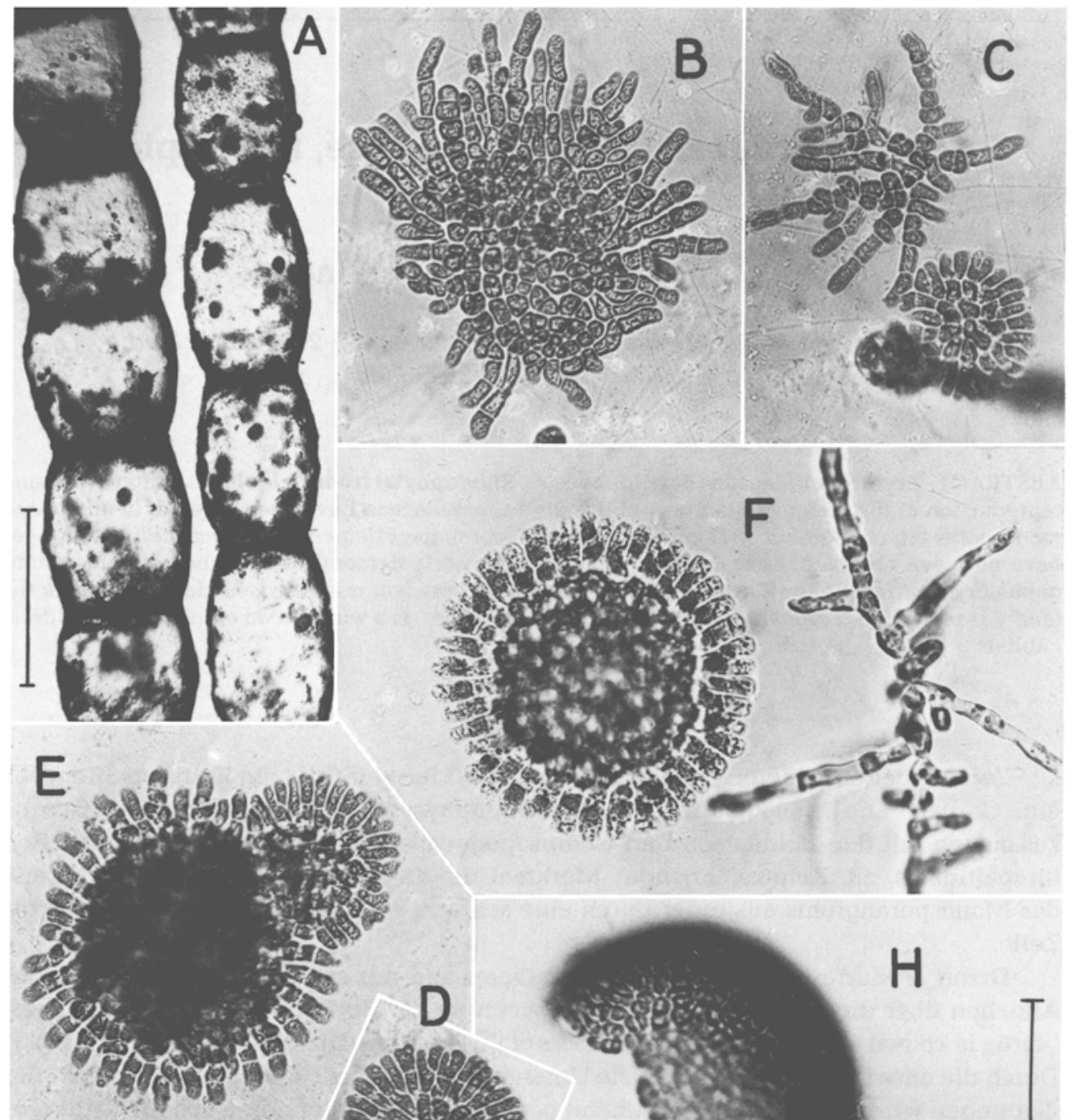
III. Thallus flächig auf einer Erythrocladia-artigen Primärscheibe

Porphyropsis

B. Lebenszyklus heteromorph

Ungeschlechtliche Phase peltoid, geschlechtliche Phase trichoid auf einer primären Basalscheibe

Erythrotrichopeltis

\section{MATERIAL UND METHODE}

Ausgewachsene Thalli von Porphyropsis coccinea oder der beiden ErythrotrichiaArten können unmittelbar von ihren Wirtsalgen isoliert werden. Sie treten aber häufig auch in Rohkulturen mit Algen oder Hydroiden aus dem Sublitoral auf, auf denen ihre Jugendstadien zusammen mit vielen mikroskopischen Epiphyten vorkommen. Auf durchsichtigen Hydroiden oder ausgeschwärmten Chaetomorpha-Fäden sind solche grünen, braunen und roten Aufwüchse zu erkennen (Abb. $1 \mathrm{~A}$ ). Ihre unmittelbare Identifizierung wäre schwierig; einfach und sicher werden sie an ihren Nachkommen erkannt. In $\mathrm{GeO}_{2}$-haltiger Nährlösung werden die Epiphyten, wenn sie es nicht schon waren, rasch fertil. Nach etwa 14 Tagen haben sich die scheibenförmigen Erythropeltidaceen auf dem Boden der Kulturschalen gut entwickelt. Abbildung $1 \mathrm{~B}$ zeigt eine schon fertile, $1 \mathrm{C}$ eine jüngere Scheibe von Erythrocladia irregularis. Die ganzrandigen Scheiben bei C, D und E sind Erythropeltis subintegra. Die großzellige Scheibe neben dem Braunalgenkeimling (F) ist Erythrotrichopeltis. Jugendstadien von Porphyropsis sind an ihrer aufgewölbten Mitte zu erkennen ( $E$ und $G$ ). Aus einem scheibenförmigen Stadium auf Chaetomorpha hat sich nach 16 Tagen ein flächiger Porphyropsis-Thallus entwickelt (H).

Isolate aus Rohkulturen wurden unter den seit vielen Jahren bewährten Bedingungen kultiviert: $15^{\circ} \mathrm{C}, 14$ stündige Beleuchtung bei Lichtstärken zwischen 1000 und 1800 Lux, je nach der Entfernung von der Leuchtstoffröhre. Als Medium diente im allgemeinen Nährlösung nach Provasoli mit einem Zusatz von Erdabkochung. Ohne erkennbare Ursache wird die Wuchsform der kultivierten Arten häufig stark durch das Substrat beeinflußt, dies gilt bei der Verwendung von Glas- oder von Plastikschalen. Parallelkulturen sind daher notwendig, um Thalli von "normaler" Erscheinungsform zu erzielen. Im allgemeinen treten solche Unregelmäßigkeiten in den Rohkulturen nicht auf; wahrscheinlich wirken sich das eingebrachte Material sowie die rasch sich vermehrenden grünen, braunen und blaugrünen Algen förderlich auf das Gedeihen aus, bevor sie die interessanten Formen überwuchern.

\section{KULTURVERSUCHE}

\section{Erythrotrichia Areschoug 1850}

Die Gattung ist bei Helgoland mit zwei Arten vertreten. Die Fäden von Erythrotrichia carnea sind über ihre ganze Länge einreihig. Die nur selten nahe der Basis

Abb. 1. A Ausgeschwärmte Zellen von Chaetomorpha melagonium mit mikroskopischen Epiphyten. B-G Thalli vom Boden etwa 14 Tage alter Rohkulturen: B, C Erythrocladia irregularis und Erythropeltis subintegra. D, E, G Erythropeltis subintegra und Porphyropsis coccinea. F Erythrotrichopeltis ciliaris, Scheibenstadium, rechts ein Braunalgen-Keimling. H Porphyropsis coccinea, auf einem Chaetomorpha-Faden nach 16 Tagen gewachsen. Maßstrecken: $A=1 \mathrm{~mm} ; \mathrm{B}-\mathrm{H}=50 \mu \mathrm{m}$ 
verzweigte Erythrotrichia reflexa ist meistens sichelförmig gekrümmt, ihr mittlerer Abschnitt ist polysiphon. Naturmaterial der beiden Arten ist in Abbildung 148 unserer "Meeresalgen von Helgoland" (1977) dargestellt:

\section{Erythrotrichia carnea (Dillw.) J. Ag.}

Kulturversuche mit $E$. carnea führen häufig nicht zu dem erwarteten Ergebnis. Trotz gleichartiger Versuchsbedingungen entwickeln sich in den Reinkulturen nur selten lange monosiphone Fäden, meistens werden sie schon frühzeitig kraus, und aus vielen Zellen sprossen kurze Verzweigungen aus (Abb. 2B). Entsprechende Beobachtungen machten auch Dixon \& West (1967) an drei Kulturen verschiedenen Ursprungs; einmal wurde diese Abnormität auch an Naturmaterial gefunden.

Eine unserer Abbildung $2 \mathrm{~B}$ ganz ähnliche Wuchsform erhielt auch Heerebout (1968, Fig. 17a) in seinen Kulturen; in der Legende wird sie als "Conchocelis stage with reduced sterile part and well-developed fertile part, producing monospores" (p. 146) gedeutet; es fehlt dafür aber jede Begründung.

Fäden von normalem Aussehen - einreihig und ohne jegliche Verzweigung - erhält man am sichersten in Rohkulturen, in denen sich $E$. carnea neben anderen Epiphyten häufig auf Algen wie Ceramium spontan entwickelt. Hier können die Fäden, wenn sie vegetativ bleiben, 4 bis $5 \mathrm{~cm}$ lang werden. In frischer Nährlösung entleeren sie schon nach 2 Tagen große Mengen von Monosporen. Die Fäden sind 14 bis $18 \mu \mathrm{m}$ breit, ihre Enden verjüngen sich auf $10 \mu \mathrm{m}(\mathrm{Abb} .2 \mathrm{~A})$.
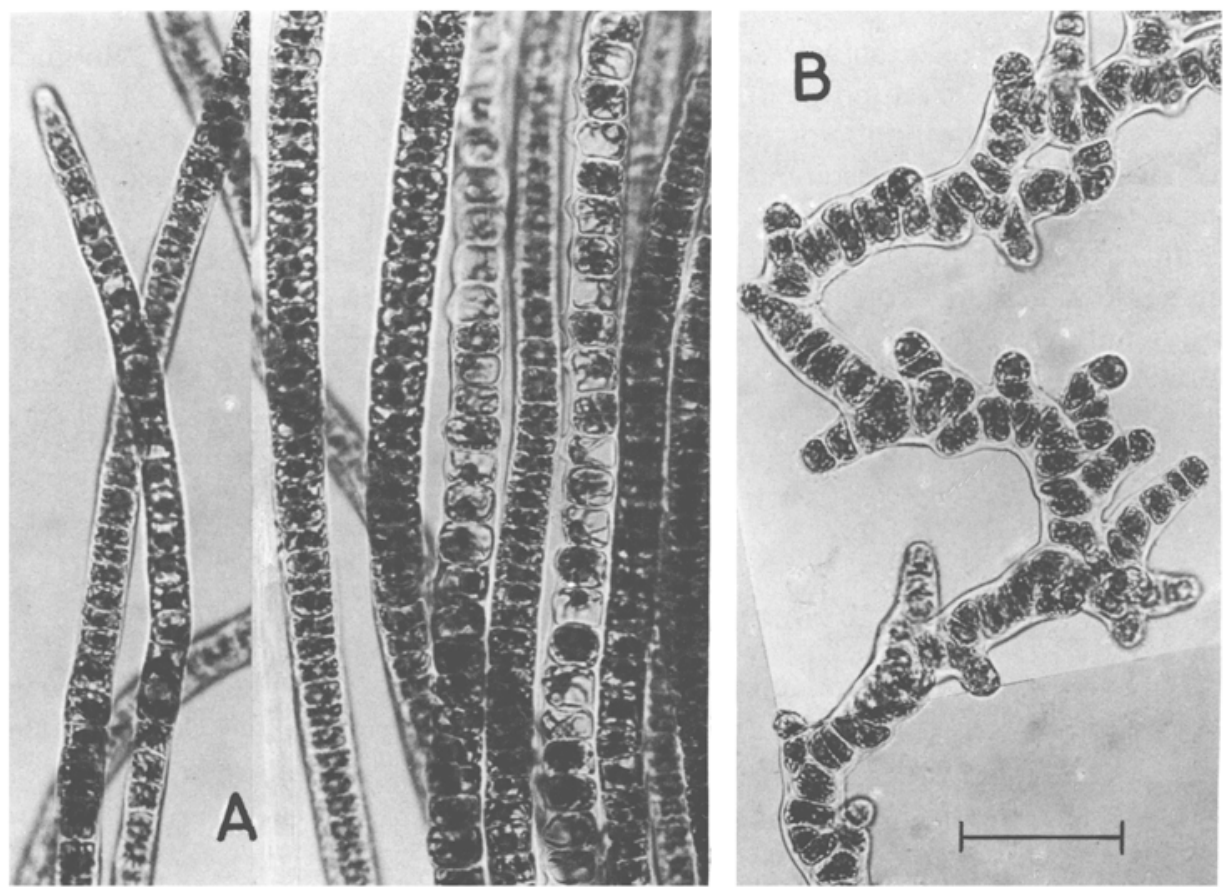

Abb. 2. Erythrotrichia carnea. A Vegetative und fertile Fäden von normalem Habitus aus einer Rohkultur. B Häufig in Kulturen vorkommende verzweigte Wuchsform. Maßstrecke: A, B $=50 \mu \mathrm{m}$ 

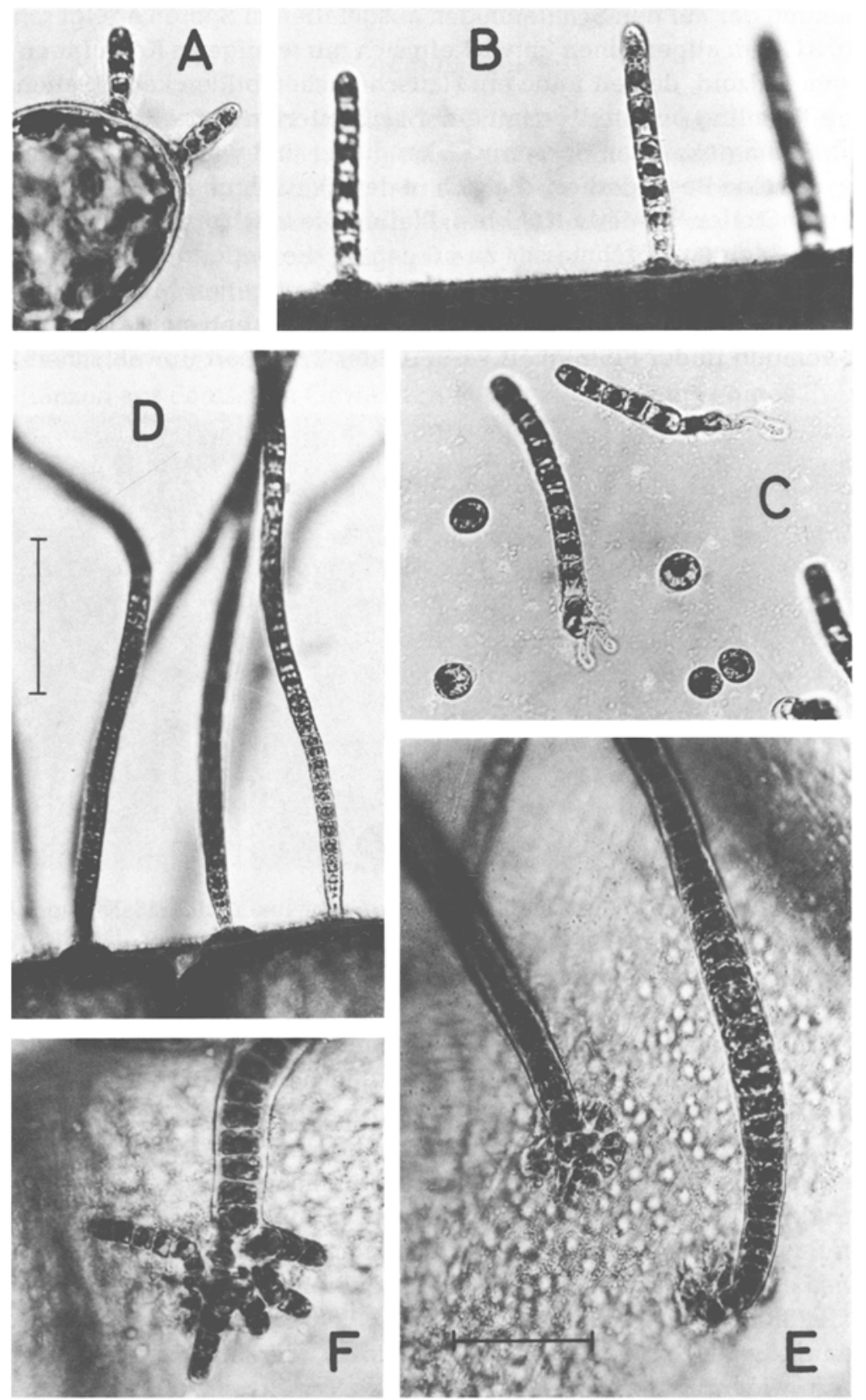

Abb. 3. Erythrotrichia carnea. A, B Zwei und vier Tage alte Keimlinge auf Bryopsis bzw. Chaetomorpha linum. B, C Aus derselben Kultur: 4 Tage alte Keimlinge auf einem Chaetomorpha-Faden und auf dem Schalenboden. D, E 10 bzw. 12 Tage alte Keimlinge. F Sekundär entstandene Haftscheibe mit jungen Fäden. Maßstrecken: $A-C, E, F=50 \mu \mathrm{m} ; D=100 \mu \mathrm{m}$ 
Die Keimung der auf den Schalenboden ausgefallenen Sporen erfolgt sehr unregelmäßig (Abb. $3 \mathrm{C}$ ). Im allgemeinen entwickeln sich nur wenige zu Keimlingen mit einem fadenförmigen Rhizoid, dessen Ende ein Haftscheibchen bilden kann. Selten haftet der wenigzellige Keimling unmittelbar mit einer verbreiterten Fußzelle auf totem Substrat: Die nicht zu Fäden gekeimten Sporen werden dicker und wandeln sich unmittelbar zu Sporangien um, eine Besonderheit, die sich in dem Ausschnitt einer Kultur während 19 Stunden an vier Stellen wiederholt (Abb. 4, Pfeile). Die frisch ausgetretenen Sporen sind leicht an ihrem kleinen Durchmesser zu erkennen, die entleerten einzelligen Sporangien an ihrem lockeren Chromatophor und der vorgezogenen Mündung. Die kleinen Monosporen können ihre Lage verändern; das Objektiv blieb stets eingetaucht, so daß ein durch Strömung in der Flüssigkeit verursachter Transport unwahrscheinlich ist.
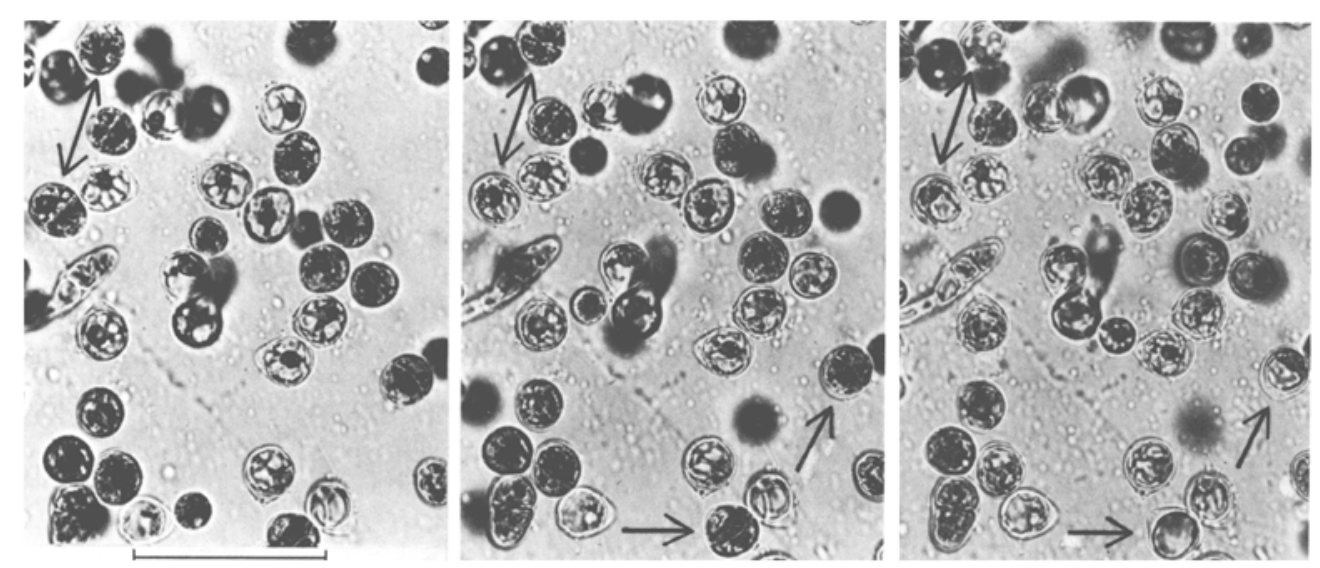

Abb. 4. Erythrotrichia carnea. Ausschnitt aus einer Kultur um 14.00, 16.00 und 8.30 Uhr des nächsten Tages. Unmittelbare Entwicklung von Sporen zu Monosporangien: Die Pfeile weisen auf solche Stadien hin. Maßstrecke $=50 \mu \mathrm{m}$

Die besonders enge Beziehung von E. carnea zu ihrer Wirtspflanze zeigt sich in eindrucksvoller Weise an dem Keimungsverhalten der Sporen. Abbildung $3 \mathrm{~B}$ und $\mathrm{C}$ entstammen derselben, vier Tage alten Kultur: alle auf den Chaetomorpha-Fäden haftenden Sporen bilden sofort eine verbreiterte Basalzelle aus (B), während sie auf dem Schalenboden nicht auskeimen oder ein fädiges, einfaches oder geteiltes Rhizoid bilden (C). Die Basalzelle der Epiphyten wächst erst nach mehreren Tagen rhizoidartig aus, und diesem Basallager entsprossen adventive Fäden (Abbildung $3 \mathrm{D}-\mathrm{F}$ ). Fadenbüschel von $E$. carnea entstehen also nicht aus einer primären Haftscheibe. Die junge Pflanze, die Dixon (1973, Fig. 5 I, nach Smith, 1944) abbildet, kann daher nicht E. carnea sein.

Das charakteristische Merkmal von E. carnea, der monosiphone, unverzweigte Faden, wurde eigentlich niemals angezweifelt. Verwirrung stiftete erst Heerebout; indem er der Art auch Fäden mit längsgeteilten Zellen und polysiphone Formen zuführte (1968, Fig. 9-11). Es erübrigt sich, seine auf dieser nicht zutreffenden Voraussetzung begründete Liste synonymer Arten zu diskutieren. 


\section{Erythrotrichia reflexa (Crouan) Thuret ex De Toni}

\section{Allgemeines}

Erythrotrichia reflexa, durch die Brüder Crouan im Jahre 1852 beschrieben, wurde erst wieder von Rosenvinge (1931) eingehend untersucht; seine Darstellung ist auch in dem inzwischen vergangenen halben Jahrhundert die einzige Grundlage geblieben. In der Diagnose der Brüder Crouan werden als wesentliches Merkmal der Art kurze, nahe der Basis entspringende Zweige angegeben; Hamel (1924; Fig. I, 4) hat sie nach Originalmaterial dargestellt. Indessen erkannte Rosenvinge die meisten Exemplare dieses Materials als unverzweigt, und in einer von Bornet bei Biarritz gesammelten Probe fand er keine verzweigten Fäden.

Die Pflanzen aus dänischen Gewässern waren mit Ausnahme eines Exemplars (Fig. 613) unverzweigt und größer als die französischen. Sie waren bis zu $5 \mathrm{~mm}$, im allgemeinen jedoch $2 \mathrm{~mm}$ lang. Der polysiphone Mittelteil war 32-63 $\mu \mathrm{m}$, meistens um $46 \mu \mathrm{m}$ dick. Diese Ausmaße können von den Pflanzen von Helgoland noch etwas übertroffen werden (Abb. 5 und Abb. 148 D-G in Kornmann \& Sahling, 1977). Verzweigte Pflanzen wurden in der Natur nicht gefunden, doch traten sie selten in den Kulturen auf. Junge, noch einreihige Fäden sind durch ihre Breite von etwa $30 \mu \mathrm{m}$ ohne weiteres von $E$. carnea zu unterscheiden (Abb. 5A). Die wenigen von Levring (1937) an der norwegischen Westküste gesammelten Exemplare waren in der Regel verzweigt, Sundene (1953) fand sie im Oslofjord meist unverzweigt. Gesicherte Angaben über das Vorkommen von E. reflexa im Mittelmeer liegen nicht vor; wir teilen die Ansicht Feldmanns (1939), der die von Dangeard (1932; Fig. 2) bei Banyuls gesammelte Pflanze nicht mit Erythrotrichia reflexa identifiziert.

\section{Kulturversuche}

Ausgangsmaterial für die Kulturen waren mehrere von sublitoralen Rotalgen isolierte Einzelpflanzen. Es sei im voraus bemerkt, daß der charakteristische Habitus des Naturmaterials - kurze, sichelförmig gebogene Thalli - nicht reproduziert werden konnte. Vielmehr wurden die Fäden in den Kulturen oft mehr als $5 \mathrm{~cm}$ lang. Zunächst waren sie nur stellenweise knotig verdickt, später wurden auch längere Abschnitte polysiphon (Abb. 6D-F).

Die Keimung erfolgt ganz entsprechend den bei Erythrotrichia carnea gemachten Beobachtungen. Auf dem Boden der Kulturschale keimt nur ein Teil der Sporen zu Fäden, meistens mit einem langen Rhizoid. Die übrigen können dicker und unmittelbar zu Monosporangien werden. Auf Chaetomorpha linum keimen alle Sporen zu Fäden, schon der zweizellige Keimling hat eine sockelartige Basalzelle (Abb. $6 \mathrm{~A}-\mathrm{C}$ ). Sporen und Keimlinge sind dicker als die von E. carnea.

Die europäischen Florenlisten führen außer den beiden gut bekannten und häufig gefundenen Erythrotrichia-Arten noch einige an, von denen wenig mehr als der Name bekannt ist. Sie genauer an Hand von einwandfrei definiertem Ausgangsmaterial zu studieren, wäre sehr wünschenswert. Als Beispiel sei die vielfach als parasitisch angesehene Erythrotrichia welwitchii genannt. Die kurzfädige verzweigte Art überzieht Ralfsia verrucosa, aber nur Thalli, die Schnecken in der Gezeitenzone besiedeln. Das Haftorgan der Rotalge dringt $z$ wischen die Fäden der Wirtsalge ein. Boney (1964) stellte fest, daß sich die Sporen von E. welwitchii auf Glas in wenigen Tagen zu 5-7 Zellen langen 

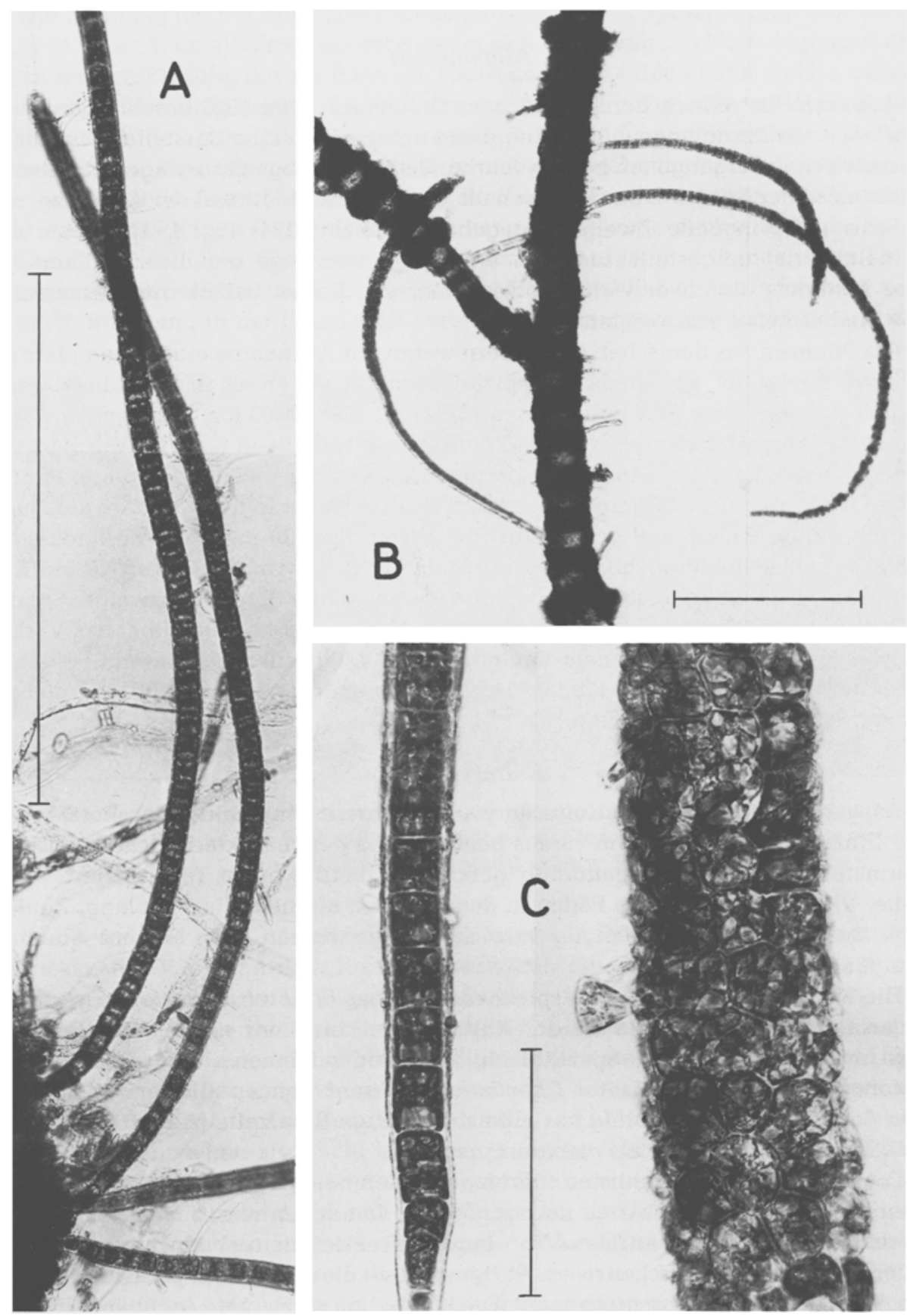

Abb. 5. Erythrotrichia reflexa, Naturmaterial. A Junge, noch monosiphone Fäden. B Sichelförmig gekrümmte Epiphyten auf Ceramium deslongchampsii. C Basis und breitester Abschnitt eines Fadens. Maßstrecken: A, B: jeweils $500 \mu \mathrm{m}_{\mathrm{i}} \mathrm{C}=100 \mu \mathrm{m}$ 
Fäden entwickeln. Planmäßige Kulturversuche versprechen eine Klärung der Taxonomie dieser Art.

Keinen Fortschritt brachte dagegen eine Untersuchung von Dangeard (1968), in der vier neue Erythrotrichia-Arten nur auf der Grundlage von Pflanzen beschrieben werden, die mehr oder weniger zufällig in seinen Kulturen aufgetreten waren. Von gut definiertem Naturmaterial auszugehen, ist eine unabdingbare Voraussetzung.

\section{Erythrocladia und Erythropeltis}

\section{Allgemeines}

Seit der Beschreibung der Gattung Erythrocladia durch Rosenvinge (1909) ist nur wenig bekannt geworden, was unsere Kenntnis von dieser Gattung wesentlich bereichert hätte, wenn auch Erythrocladia irregularis und Erythrocladia subintegra häufig gefunden und abgebildet worden sind. Durch zahlreiche Arten aus aller Welt vermehrt, wurde die Gattung zu einem Sammelbecken der verschiedensten Formen (Übersicht bei Nichols \& Lissant, 1967). Es ist daher zweckmäßig, unmittelbar an die Ausführungen Rosenvinges (1909) über drei dort diskutierte scheibenförmige Arten anzuknüpfen.

Die Gattung Erythrocladia ist durch ihre Diagnose unmißverständlich gekennzeichnet; das wesentliche Merkmal ist der Zusammenschluß apikal wachsender Fäden mit seitlicher Verzweigung zu einer Scheibe. Diesem Merkmal der typischen Art, Erythrocladia irregularis, fügt sich Erythrocladia subintegra nicht ein. Hier besteht nämlich die "Verzweigung" in einer dichotomen Spaltung der Endzellen, so daß von Anfang an eine geschlossene Scheibe mit Randwachstum entsteht. Auf einen derartigen Thallus hatte Schmitz (1896) seine Gattung Erythropeltis begründet mit der von Berthold (1882; Tafel 1, Fig. 15 und 16) abgebildeten Scheibe von Erythrotrichia discigera als typischer Art. Rosenvinge hatte zwar die Ähnlichkeit seiner Erythrocladia subintegra mit dieser Scheibe und mit der von Batters (1900) beschriebenen Erythropeltis discigera var. flustrae erkannt, deren Zellen und Sporen aber wesentlich größer sind als die von Erythrocladia subintegra. Auch schlossen die von ihm häufig als getrennt angesehenen Endzellen von Erythrocladia subintegra die Eingliederung in die Gattung Erythropeltis aus. Das getrocknete Material, das Rosenvinge vorlag - Epiphyten auf Polysiphonia urceolata aus 11-15 m Tiefe -, ließ den Aufbau der Scheiben nur unvollkommen erkennen.

1915 bildete Børgesen auf Chaetomorpha und Cladophora gefundene Scheiben von Erythrocladia subintegra ab. Völlig zutreffend wird der Aufbau des Thallus beschrieben. "It has marginal growth and, as pointed out by Rosenvinge, the cells are dichotomously divided but the bifurcation is most often not quite equal, the one cell being larger than the other. Near the margin the cells are often linear-oblong or forked, in the middle shorter, oblong, or of more irregular shape" (p. 8). Nur mit Vorbehalt beließ Børgesen die Art bei Erythrocladia; ihre Eingliederung in die Gattung Erythropeltis Schmitz erschien ihm aus heute nicht mehr aktuellen Gründen nicht vertretbar.

Nachdem Erythrocladia irregularis und E. subintegra so häufig gefunden und abgebildet worden sind, ist es verwunderlich, daß Børgesens Zweifel niemals dazu führte, sie verschiedenen Gattungen zuzuordnen. Im Gegenteil: Heerebout (1968) erkannte nicht einmal die Selbständigkeit der beiden Arten Rosenvinges an. "Culture 

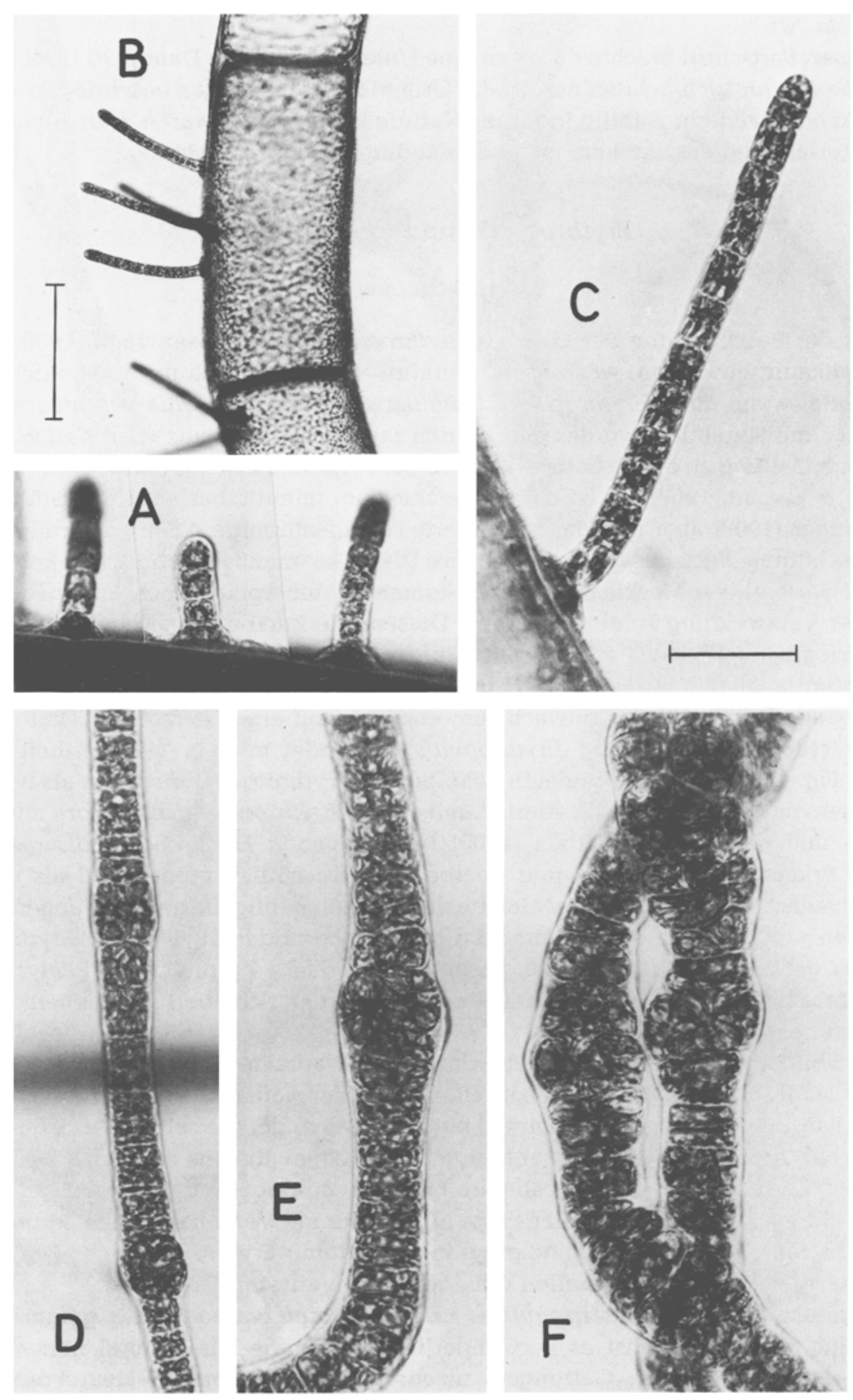
experiments showed that Erythrocladia irregularis, E. subintegra and E. polystromatica are only forms of one species" (p. 141). Wie die Figuren 1 bis 4 zeigen, war das Untersuchungsmaterial, das dieser Angabe zugrunde liegt, nicht einheitlich.

\section{Erythrocladia irregularis Rosenvinge}

Die Diagnosen und die Abbildungen Rosenvinges (1909) kennzeichnen diese Art als monostromatische, auf anderen Algen haftende Scheibe von unregelmäßigem Umriß. Sie entwickelt sich aus einem nach beiden Richtungen apikal wachsenden Fädchen, das sich beiderseitig wiederholt verzweigt. Die zunächst getrennten, radiär sich ausbreitenden Fäden vereinigen sich in der Mitte zu einer pseudoparenchymatischen Scheibe; die Fadenenden sind frei. Nach 8 bis 10 Tagen werden die zentralen Zellen fertil; die Monosporen sind ziemlich einheitlich etwa $5 \mu \mathrm{m}$ dick. Abbildung 7 illustriert diese Beschreibung zur Genüge.

Der Habitus von Erythrocladia irregularis findet bei den Grünalgen sein genaues Ebenbild in Stromatella monostromatica (Dang.) Kornm. et Sahling (1983), der typischen Art dieser Gattung*. Die beiden Taxa stimmen sogar in der durch das Substrat bedingten Variabilität ihrer Wuchsform überein. Bei einer größeren Anzahl von Kulturen - sei es in Plastikschalen oder auf Deckgläsern - beobachtet man stets auch Scheiben von einer gedrungenen Wuchsform und kürzeren Zellen, ohne daß eine Ursache dafür erkennbar wäre (Abb. 7 C). Der "unnatürliche" Habitus solcher Formen ist jedoch nicht zu übersehen. Auf älteren Pflanzen erheben sích auch einzelne Fäden frei in die Flüssigkeit (D).

E. irregularis wurde häufig zusammen mit E. subintegra an den Küsten der Nordund Ostsee beobachtet; sie dringt aber offenbar nicht wie $E$. subintegra in wärmere Meere vor. Sie fehlt in der Algenflora von Roscoff (Feldmann, 1954), ist für die Küsten Englands bei Newton (1931) nicht angegeben, wohl aber bei Parke (1953).

\section{Erythropeltis Schmitz 1896}

Die Gattung Erythropeltis wurde von Schmitz (1896; p. 313) auf der scheibenförmigen Erscheinungsform von Erythrotrichia discigera Berthold begründet. Als Phase im Entwicklungszyklus von Erythrotrichopeltis ciliaris (Kornmann, 1984) wäre die Gattung eigentlich hinfällig, wenn nicht die Diagnose von Schmitz die Merkmale der ihr hier zugeführten Erythrocladia subintegra kennzeichnete.

* Die Diagnose der Gattung Stromatella in Kornmann \& Sahling (1983; p. 10) ermangelt der Angabe der typischen Art. Species typica ist Stromatella monostromatica (Dangeard), nov. comb. Basionym: Ulvella monostromatica Dangeard, 1965, p. 45, pl. I, fig. 8-13. pl. III, fig. 1.

Der Gattung wurde außerdem zugeführt Stromatella papillosa (Dangeard) nov. comb. (Kornmann \& Sahling, 1983; p. 12). Basionym: Ulvella papillosa (Dangeard 1965, p. 50, pl. II, fig. 1-6, pl. IV, fig. 2; 1969 , p. 42 , pl. VI und X.

Abb. 6. Erythrotrichia reflexa. A-C Keimlinge auf Chaetomorpha linum, 2 und 5 Tage alt. D 13 Tage alter Faden mit regelmäßig wiederkehrenden Längsteilungen in einzelnen Zellen. E, F Altere, polysiphone Fadenstücke. Maßstrecken: $A, C-F=50 \mu \mathrm{m} ; B=200 \mu \mathrm{m}$ 


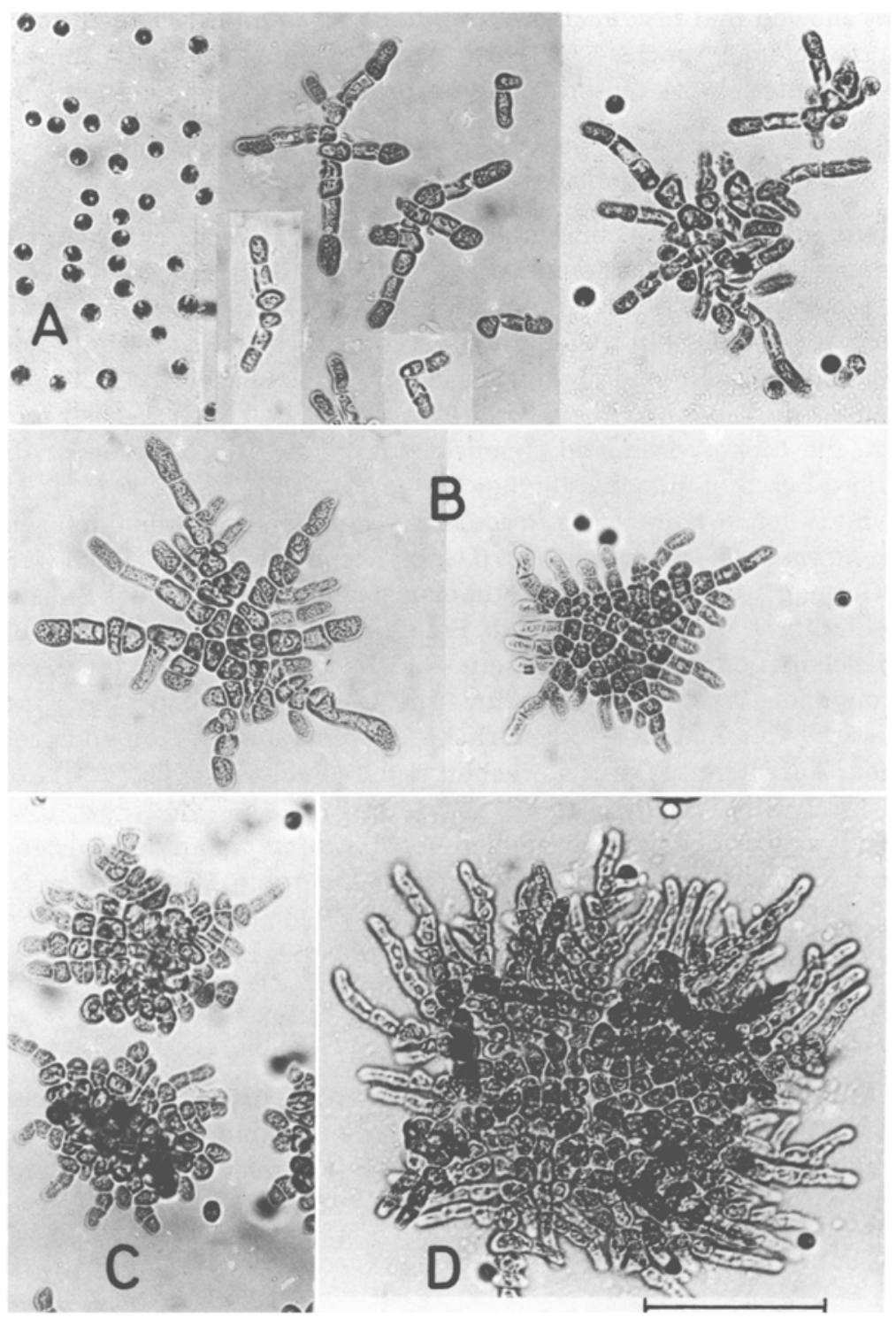

Abb. 7. Erythrocladia irregularis. A Sporen, bis zu 22 Stunden alt. B Thalli verschiedenen Alters und verschiedener Herkunft. C Gedrungene Wuchsform. D Älterer Thallus mit frei in die Flüssigkeit wachsenden Fäden. Maßstrecke: $A-D=50 \mu \mathrm{m}$

Erythropeltis subintegra (Rosenv.) nov. comb.

Basionym: Erythrocladia subintegra Rosenv. 1909, p. 73, Figs 13 und 14

Synonym: Erythrocladia gibber Dangeard, forme à petites cellules marginales, 1968 , p. 19, Pl. I, Figs 1-8, PI. VII, C, D, Pl. VIII, Pl. IX, A, D. 
Nach Rosenvinges Beschreibung ist der Thallus von Erythrocladia subintegra mehr rundlich als der von Erythrocladia irregularis, und die Fäden strahlen allseitig ziemlich regelmäßig aus. Meist sind sie fest bis zu den Enden seitlich miteinander verwachsen, und nur die Endzellen sind mehr oder weniger getrennt. Die Verzweigung erfolgt ausschließlich oder hauptsächlich in den Endzellen, die sich gabelig teilen, wobei eine der Schwesterzellen stärker sein kann als die andere.

Der Diagnose Rosenvinges lag getrocknetes Material zugrunde, dennoch wurden die wesentlichen Merkmale klar erkannt. Børgesens Beschreibung (1915) und Abbildungen vervollständigten die Kennzeichnung dieser Alge, deren Ontogenese in Abbildung 8 A dargestellt ist. Zwei Tage alte Keimlinge sind meist zweizellig, aus jeder Zelle stülpen sich ein oder zwei hyaline Auswüchse vor. Die nächste Teilung führt zu vier herzförmig ausgebuchteten Zellen, die sich kreuzweise zu einer kleinen Scheibe zusammenschließen. Am folgenden Tag hat eine schräge Wand jeden Sektor in ungleiche Zellen geteilt: der längeren, bis ins Zentrum reichenden Zelle sitzt eine kürzere seitlich auf. Dieses Teilungsschema wird von den stark sich streckenden und dichotom sich teilenden Randzellen beibehalten. Sie säumen die isodiametrischen Zellen der Scheibenmitte. 8 bis 9 Tage alte Scheiben werden fertil. Die Monosporen sind durchschnittlich $6 \mu \mathrm{m}$ dick (Abb. 8B). Dünne Algen oder Baumwollfasern werden von den Scheiben mantelförmig umhüllt (Abb. $8 \mathrm{C}$ ); im optischen Schnitt sind die Zellen in der Scheibenmitte etwa $8 \mu \mathrm{m}$ hoch. In gleicher Weise wie Erythropeltis subintegra entwickelt sich die peltoide Phase von Erythrotrichopeltis, deren Zellen und Sporen aber wesentlich größer sind (Kornmann, 1984).

Wie sich Erythrocladia irregularis und Stromatella monostromatica morphologisch gleichen, so entwickeln sich auch die Scheiben von Erythropeltis subintegra und von Ulvella lens nach dem gleichen Muster (Kornmann \& Sahling, 1983; Abb. 9).

Nachdem Dangeard (1932) Erythrocladia subintegra nach Naturmaterial dargestellt hatte, beschrieb er 1968 dieselbe in seinen Kulturen aufgetretene Alge neu unter dem Namen Erythrocladia gibber; er unterschied zwei Formen nach der Größe der Randzellen. Die "forme à petites cellules marginales" entspricht Erythropeltis subintegra, die "forme à grandes cellules marginales" ist mit dem scheibenförmigen Thallus von Erythrotrichopeltis ciliaris identisch (Kornmann, 1984). Die zahlreichen Zeichnungen und Photographien Dangeards lassen an diesen Zuordnungen keinen Zweifel. In den Kulturen treten häufig auch Scheiben mit höckerigen Auswüchsen oder knäuelig gewachsene Thalli auf (Abb. 8D), eine Besonderheit, die Dangeard durch das Epithet zum Ausdruck brachte. Ebenso neigen die Scheiben in ruhigstehenden Kulturen dazu, sich in ihrer Mitte kissenartig aufzuwölben, weil die Monosporen nicht wie in dem natürlich bewegten Medium weggespült werden.

Erythropeltis subintegra ist an den nördlichen atlantischen und pazifischen Küsten verbreitet, kommt aber auch in wärmeren Meeren vor, in die ihr Erythrocladia irregularis nicht folgt (Børgesen, 1915; Feldmann, 1929; Dangeard, 1932; Kapraun, 1980). Die von Smith (1944) als Erythrocladia subintegra dargestellte scheibenförmige Alge gehört weder zu Erythrocladia noch zu Erythropeltis, worauf schon Dangeard (1968, p. 18) hingewiesen hat. Die von Fritsch (1945, Fig. 144 C) irrtümlich als Erythrocladia subintegra abgebildete Alge ist in Kylins (1925) Original richtig als E. irregularis bezeichnet.

Offensichtlich nicht mit der europäischen Art identisch sind die von Nichols \& Lissant (1967) im Golf von Mexiko gesammelten und als Erythrocladia subintegra 


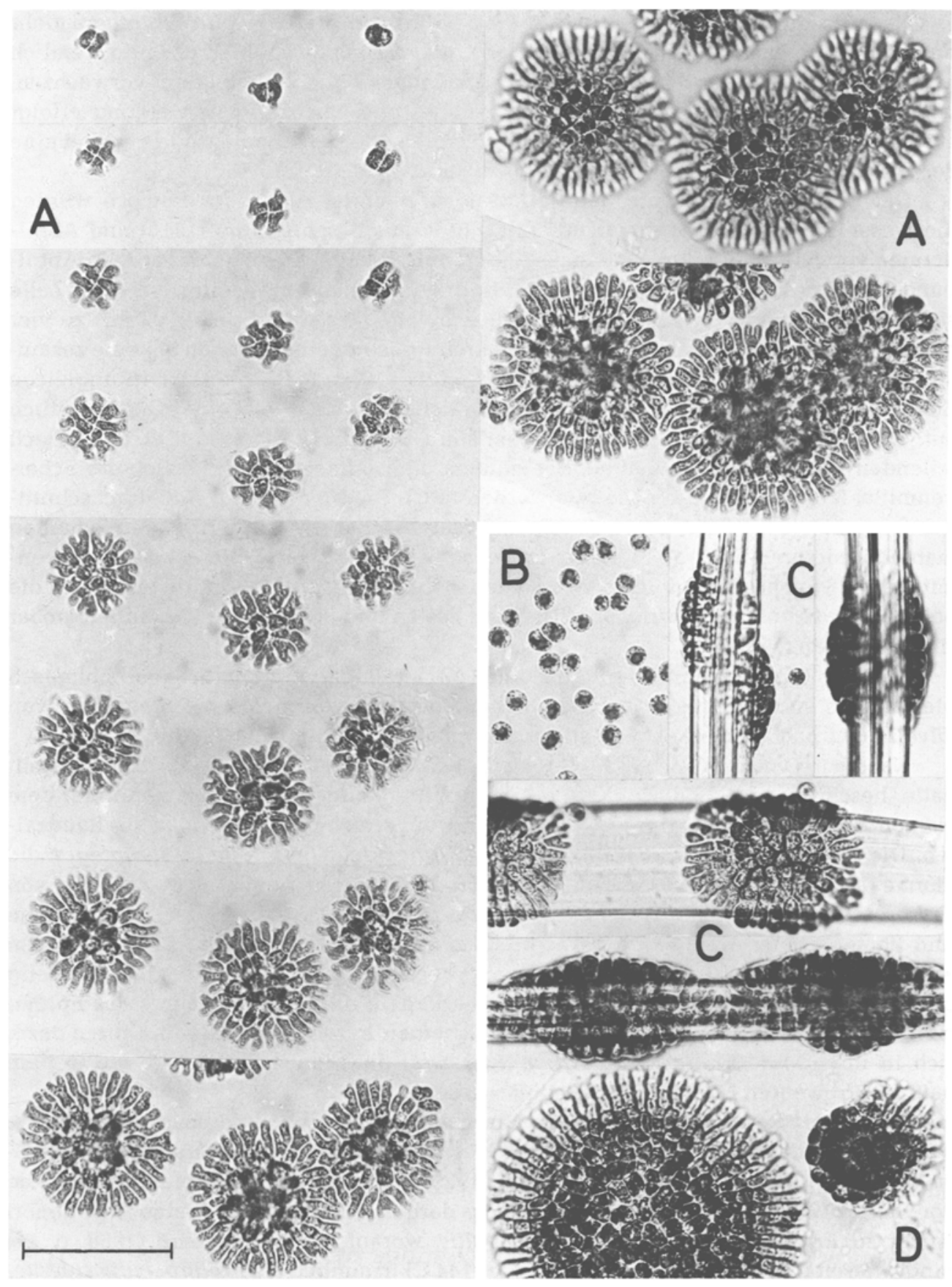

Abb. 8. Erythropeltis subintegra. A Entwicklung der Scheibe vom 2. bis zum 10. Tag, zuletzt in verschiedener Bildhöhe aufgenommen. B Sporen, bis zu 22 Stunden alt. C Thalli auf Baumwollfasern, 6, 10 und 13 Tage alt. D Aus einer 16 Tage alten Kultur, rechts ein Thallus mit Höcker. Maßstrecke: $\mathrm{A}-\mathrm{D}=50 \mathrm{~mm}$ 


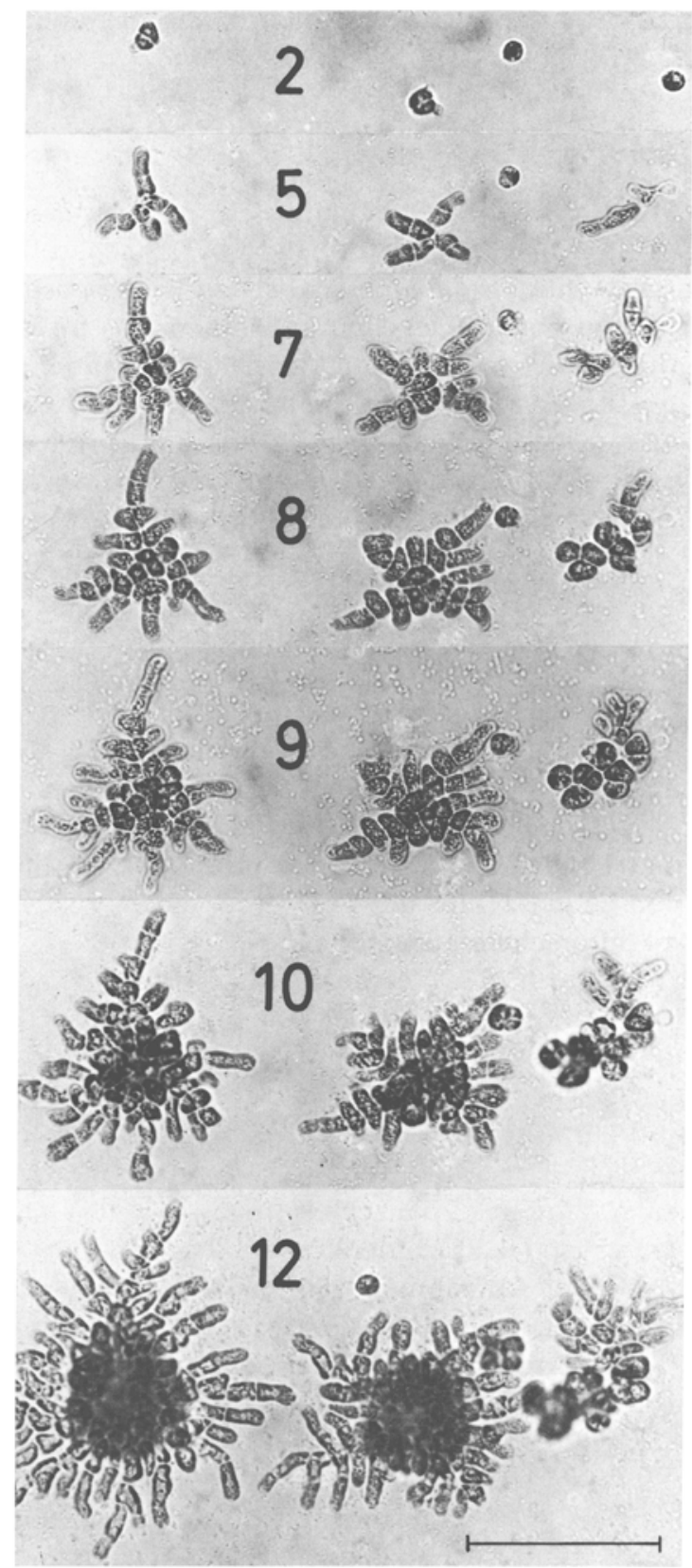

Abb. 9. Porphyropsis coccinea. Entwicklung des scheibenförmigen Thallus vom 2. bis zum 12. Tag, die Scheibenmitte beginnt sich aufzuwölben. Der Keimling rechts bildet zunächst keine auf dem Substrat haftende Scheibe, sondern ungeordnete kugelige Zellen. Maßstrecke $=50 \mu \mathrm{m}$ 
bezeichneten Scheiben. Die Arbeit wurde in anderem Zusammenhang diskutiert (Kornmann, 1984 ; p. 210).

\section{Porphyropsis coccinea (J. Ag.) Rosenvinge}

Rosenvinge (1909) beschrieb die Entstehung des monostromatischen Thallus von Porphyropsis coccinea an Hand von Naturmaterial. Danach sollte sich die oberste Zellschicht eines mehrschichtigen parenchymatischen Scheibenstadiums ablösen und durch aktives Wachstum zu einem Säckchen aufwölben, das frühzeitig aufreißen und dem blattartigen, auf einer kissenförmigen Basalscheibe angehefteten Thallus den Ursprung geben sollte. Diese in den Einzelheiten nicht ganz zutreffende Darstellung fand allgemein Eingang in die Lehrbücher, z. B. Fritsch (1945), Kylin (1956). Sie kehrt auch noch in einer Arbeit von Murray et al. (1972) wieder, welche die Entwicklung von Porphyropsis coccinea var. dawsonii im Laboratorium untersuchten. Auch hier wird eine "polystromatic disc" als Jugendstadium angegeben und mit einer wenig überzeugenden Abbildung (Fig. 16) belegt. Wie unsere Versuche zeigen, gibt es ein solches mehrschichtiges oder kissenförmiges Jugendstadium bei $P$. coccinea nicht.

Porphyropsis gedeiht am besten in Kulturen mit zerstreut liegenden Sporen. Ein Teil von ihnen entwickelt sich "normal" zu scheibenförmigen Thalli; einige keimen nicht oder nur verzögert; andere können zu unregelmäßigen, dickzelligen Fädchen auswachsen. Vereinzelt entstehen auch unmittelbar freitreibende monostromatische Porphyropsis-Flächen. In manchen Kulturen werden die scheibenförmigen Thalli frühzeitig fertil. Alle diese Abweichungen können hier unberücksichtigt bleiben; sie sind nicht regelmäßig in den Lebenszyklus der Alge eingeschaltet, sondern können nebeneinander in derselben Kulturschale auftreten.

Die Entwicklung beginnt in der gleichen Weise wie bei Erythrocladia irregularis (Abb. 9). Während der ersten 10 Tage entsteht eine unregelmäßige Scheibe aus akropetal verzweigten Fäden und einer Mitte aus polygonalen Zellen. Zwei Tage später ist die Scheibenmitte schon aufgewölbt.

Die Entstehung des flächigen Thallus ist in Abbildung 10 dargestellt. Die Bilder folgen einander in Abständen von zwei Tagen, Aufnahmen in verschiedener Bildhöhe zeigen den räumlichen Aufbau des Pflänzchens. In der Aufsicht auf die Oberfläche des 11 Tage alten Pflänzchens ist der Rhizoidenkranz schon undeutlich, am 13. Tage ist er nur schwach erkennbar. Die Aufnahmen vom 15. und 17. Tag sind zu einem Bild kombiniert, sie vermitteln den subjektiven Eindruck des mikroskopierenden Beobachters. Uber einem Rhizoidenkranz, dessen Fäden sich auf dem Substrat ausbreiten und verzweigen, wölbt sich eine immer mehr halbkugelig werdende Kuppel. Das einschichtige aufgewölbte Parenchym entsteht durch interkalare Teilung der polygonalen Zellen der Scheibenmitte. Die Bilder in der untersten Reihe setzen die Serie bei schwacher Vergrößerung noch bis zum 27. Tage fort; das Säckchen ist perforiert und fertil geworden. Vegetativ bleibende Thalli können 2 bis $2,5 \mathrm{~cm}$ groß werden; nach dem Umsetzen in frische Nährlösung werden sie rasch fertil.

Abb. 10. Porphyropsis coccinea. Entstehung des flächigen Thallus durch Aufwölbung der monostromatischen Scheibenmitte. Die Aufnahmen in verschiedenem Niveau sind am 15. und 17. Tag zu einem Bild zusammengesetzt. Maßstrecken: $50 \mu \mathrm{m}$ bis zum Alter von 19 Tagen; $100 \mu \mathrm{m}$ für die Fortsetzung in der unteren Reihe 

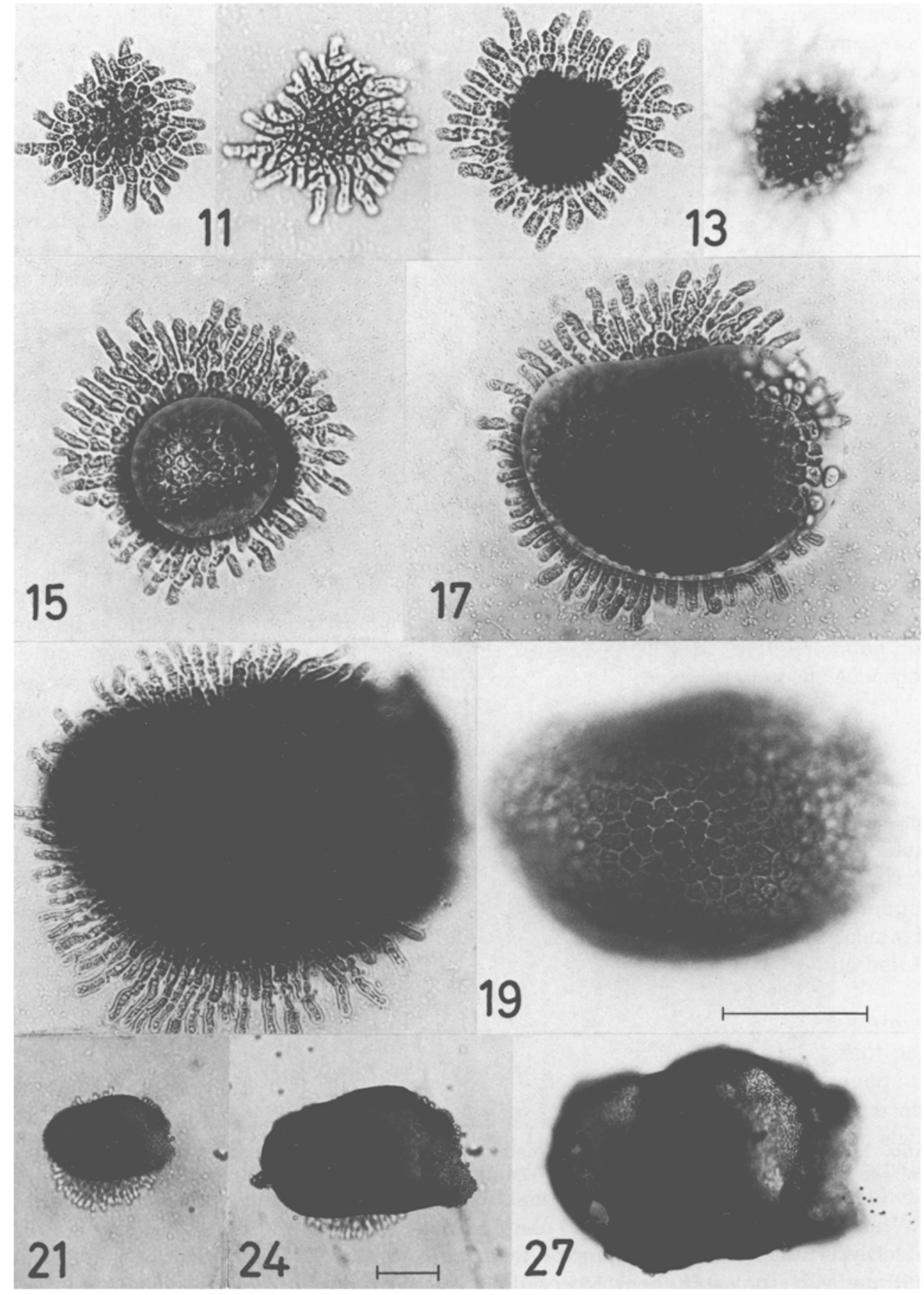

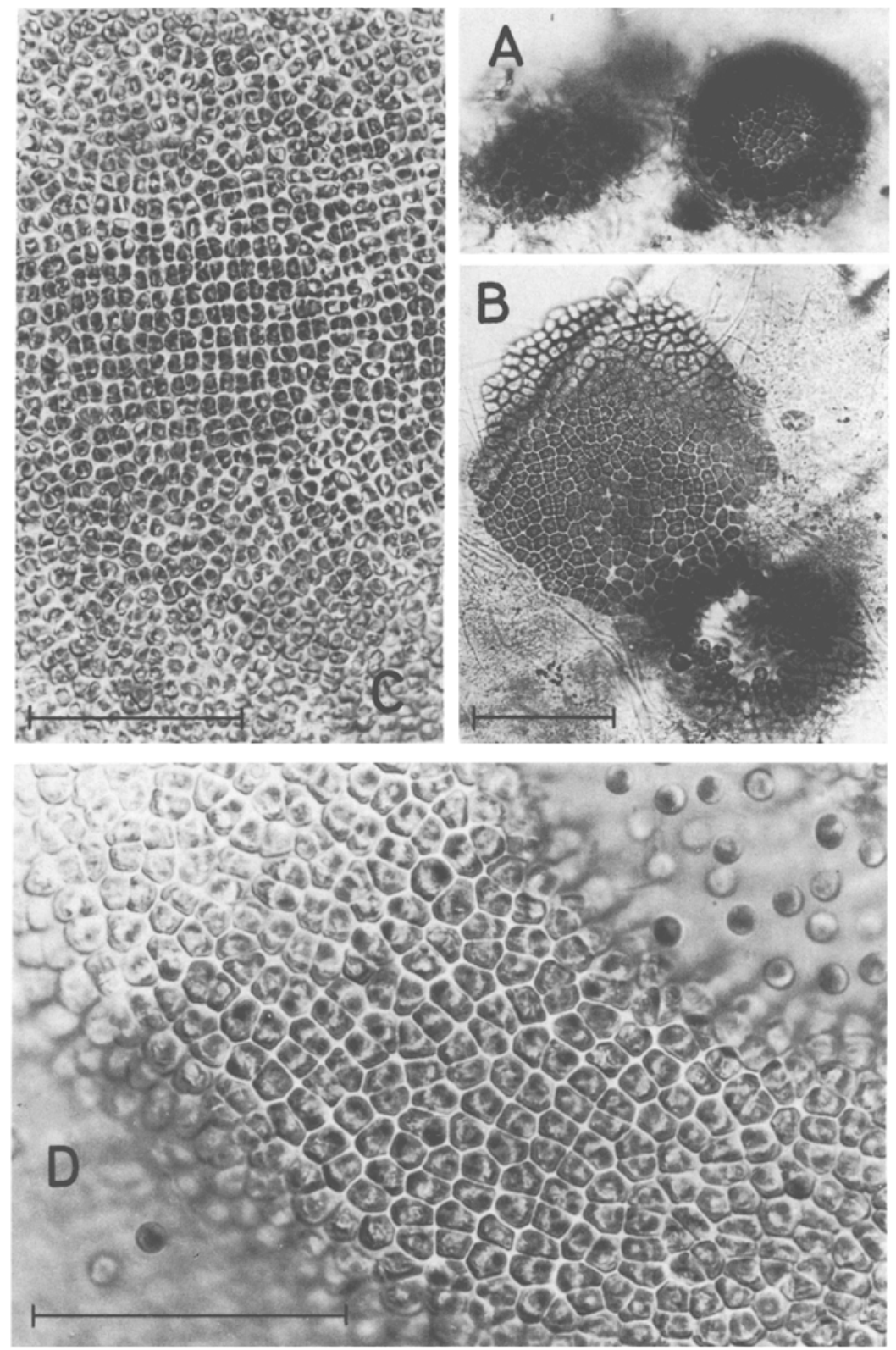

Abb. 11. Porphyropsis coccinea. Naturmaterial. A, B Entwicklungsstadien auf Chaetomorpha melagonium, 21. 4. 1977. C Vegetativer Thallus. D Fertiler Thallus mit freien Monosporen. Maßstrecken: A und B, C, D jeweils $=50 \mu \mathrm{m}$

Diese Studie über Porphyropsis coccinea wäre unvollständig ohne den Vergleich mit natürlich gewachsenem Material. Ausgeschwärmte Chaetomorpha-Fäden vom 21. 4. 1977 waren reichlich mit jungen Stadien von Porphyropsis besetzt. Um klare 
Bilder zu erhalten, wurden die Fäden zerschnitten und die Bruchstücke der Zellmembranen mit den Epiphyten ausgebreitet. Die Entwicklungsstadien im Naturmaterial (Abb. 11) gleichen ganz denen in den Kulturen. Bei A erhebt sich das noch geschlossene monostromatische Säckchen über einem Rhizoidenkranz. Diesem Ring ist das junge Blatt seitlich angeheftet (B). Die Mitte des Ringes ist noch offen, sie wird erst nachträglich durch sekundäre Rhizoiden ausgefüllt.

In dem vegetativen Thallus von $P$. coccinea sind die isodiametrischen Zellen in deutlichen, mitunter senkrecht aufeinanderstehenden Reihen angeordnet (Abb. $11 \mathrm{C}$ ); an der Basis verlängern sie sich rhizoidartig, wie es Rosenvinge (1909, Fig. 9) abgebildet hat. Frisch gesammelte Pflanzen entleeren in Nährlösung meist schon am nächsten Tag zahlreiche Monosporen; einen Ausschnitt aus einem solchen Thallus zeigt Abbildung 11 D. Die Monosporen sind sehr einheitlich $5 \mu \mathrm{m}$ dick. Andere als in den für die Ordnung typischen differenzierten Sporangien entstandene Monosporen wurden nicht beobachtet.

$P$. coccinea ist eine Alge der nördlichen atlantischen und pazifischen Küsten; nach Dizerbo (1969) erreicht sie in Europa die südliche Grenze ihrer Verbreitung bei Roscoff; das Material der Brüder Crouan stammt von der Reede von Brest (Hamel, 1924). P. coccinea von der kalifornischen Küste wurde als var. dawsonii vom Typus unterschieden. Über ihre Entwicklung haben Murray et al. (1972) berichtet.

Heerebout (1968) führte Porphyra vexillaris Montagne der Gattung Porphyropsis zu; diese Kombination ist angesichts der in seiner Figur 12 abgebildeten Basis nicht vertretbar. 1975 beschrieben Womersley \& Conway eine neue Art aus Südaustralien: Porphyropsis minuta. Die nicht dem Ordnungsmerkmal entsprechende Entstehung der Sporen - der gesamte Inhalt der Randzellen des Thallus wird unmittelbar zu Monosporen -, schließt diese Alge aus der Gattung Porphyropsis aus.

\section{Porphyropsis imperfecta nov. spec.}

In vielen Rohkulturen mit Algen oder Hydroiden aus dem Sublitoral trat eine dunkelrot bis violett gefärbte Alge auf, deren frühe Entwicklungsstadien ganz denen von Porphyropsis coccinea gleichen (Abb. 12A). Es sind Scheiben von radial sich ausbreitenden und verzweigenden Fäden mit einer Mitte aus polygonal zusammengeschlossenen Zellen. Im Alter von 10 Tagen beginnen die monostromatischen Scheiben sich deutlich aufzuwölben, sie werden dann auch reichlich fertil. Anders als bei $P$. coccinea reißt aber die Oberfläche des schüsselförmigen Thallus niemals auf.

Zu einem Vergleich unter denselben Kulturbedingungen wurden die beiden Algen getrennt voneinander in einer großen Petrischale kultiviert. Abbildung 13 zeigt das Ergebnis nach 18 Tagen: die flachgewölbten und reichlich fertilen Thalli der neuen Art (A) neben den flächigen Thalli von $P$. coccinea (B). Auf Chaetomorpha linum als Substrat bilden die Epiphyten flach aufgewölbte Thalli; 14 Tage alte Pflanzen sind etwa 25-30 $\mathrm{m}$ hoch (Abb. 12 B, C). Die gleichalten, auf dem Boden derselben Kulturschale gewachsenen Thalli sind über einem Rhizoidenkranz aufgewölbt (Abb. 12D). Schließlich mögen noch zwei Aufnahmen in verschiedenen Bildebenen die Form der neuen Art anschaulich machen (Abb. 13 C). Der vom Schalenboden gelöste und umgedrehte hohle Thallus haftet mit einem Kranz verzweigter Fäden auf dem Substrat, die flach aufgewölbte monostromatische Decke besteht aus polygonalen Zellen. Aufbau und Entwick- 


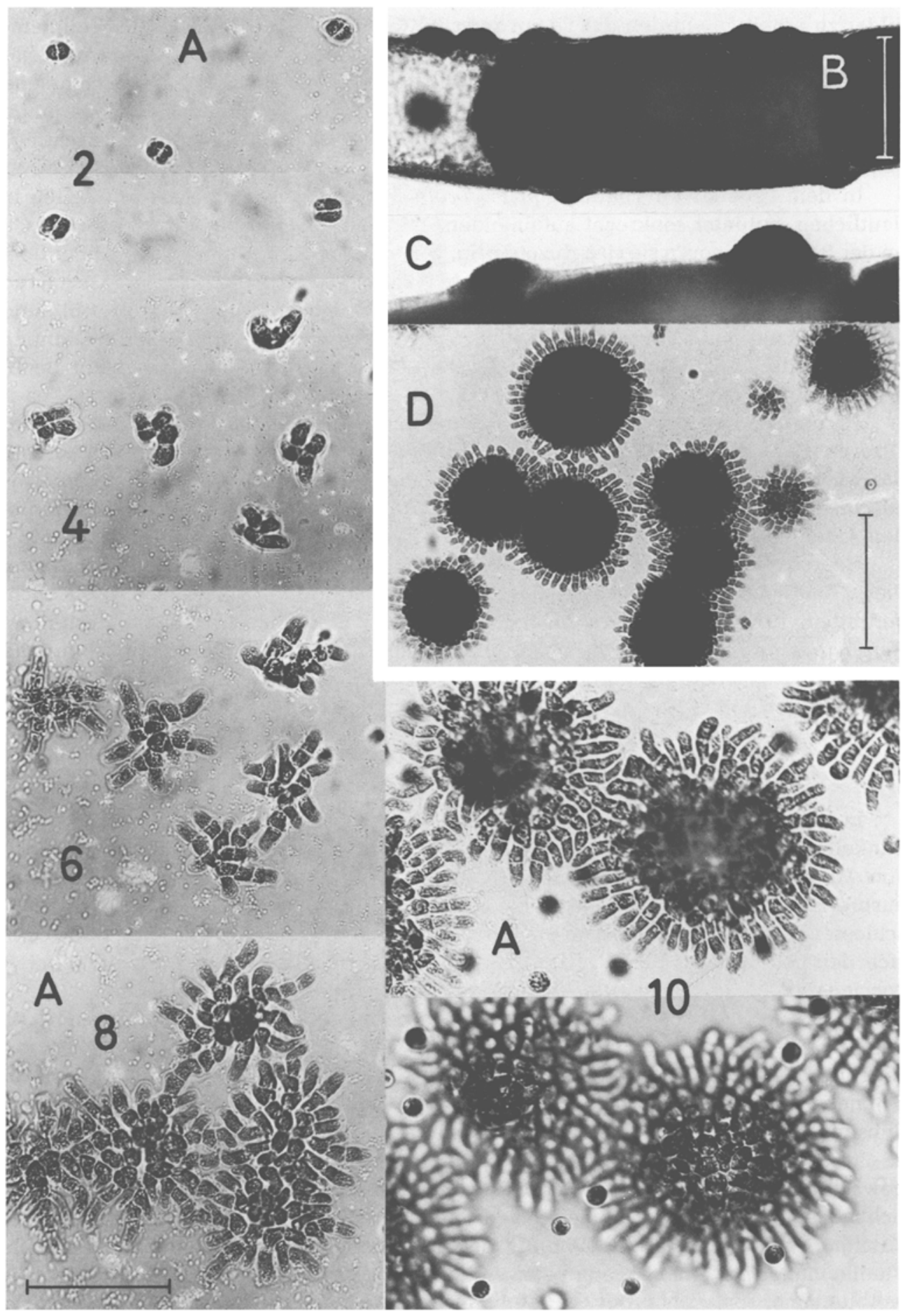


lung der Alge erreichen das Jugendstadium von $P$. coccinea, jedoch reißt die Oberfläche nicht auf wie bei der typischen Art. Das Epithet imperfecta bringt dieses unterscheidende Merkmal zum Ausdruck.

Diagnose: Der ausgewachsene Thallus von $P$. imperfecta gleicht einer mit ihrem Rand auf dem Substrat haftenden Schale von 200-300 $\mu \mathrm{m}$ Durchmesser. Der Rand besteht aus apikal wachsenden verzweigten Fäden, der gewölbte einschichtige, etwa $30 \mu \mathrm{m}$ hohe Mittelteil aus isodiametrischen Zellen. Die 6-7 $\mu \mathrm{m}$ dicken Monosporen entwickeln sich in gleicher Weise wie $P$. coccinea zu einer Scheibe; ihre Mitte wölbt sich auf, sie reißt jedoch nicht auf wie bei der typischen Art.

Herkunft: Porphyropsis imperfecta kommt häufig auf Algen und Hydroiden aus dem Sublitoral von Helgoland vor, aus deren Rohkulturen sie isoliert wurde.

Diagnosis: Frons adulta similis est catillo, qui parte marginali adhaeret substrato. Diametrum catilli circa $200-300 \mu \mathrm{m}$. Margo constat e ramosis filamentis apicaliter crescentibus; media pars convexa monostromatica, circa $30 \mu \mathrm{m}$ alta constructa est e cellulis isodiametricis. Monosporae 6-7 $\mu \mathrm{m}$ diam., formant eodem modo atque Porphyropsis coccinea discum, cuius media pars convexa non est ruptura contrarie speciei typicae.

Orig o: Species occurrit frequenter ad algas et hydroides in zona sublitorali insulae Helgolandiae.

\section{Erythrotrichopeltis ciliaris (Carm.) Kornmann}

Taxonomie und Entwicklung von Erythrotrichopeltis ciliaris waren Gegenstand einer früheren Studie (Kornmann, 1984). Die scheibenförmige Phase ihres heteromorphen Entwicklungszyklus (Abb. 1 F) - 1982 und 1983 aus Rohkulturen sublitoraler Algen isoliert - wurde nochmals am 26. August 1984 auf Hydroiden gefunden. Die mit diesem Material wiederholten Versuche führten zu dem gleichen Ergebnis. Eine mit wenigen Scheiben am 2. Oktober angelegte Kultur breitete sich über den Boden einer $4 \mathrm{~cm}$ großen Plastikschale aus. Als die Nährlösung am 8. Januar 1985 erneuert wurde, enthielt der weitgehend entfärbte Bodenbelag nur noch wenige dunkle knäuelige Zellaggregate. Aus einigen von ihnen waren schon am 27. Januar Erythrotrichia-Fädchen entsproßt. Sie wuchsen in frischer Nährlösung rasch zu fertilen Gametophyten heran. Die angewandte Versuchsmethode ist nicht elegant; wahrscheinlich wird sich der Entwicklungsablauf durch variierte Kulturbedingungen steuern lassen. Solche Versuche waren nicht vorgesehen.

Als fädige Phase ist $E$. ciliaris in der Natur bei Helgoland noch nicht gefunden worden. Ihre Entstehung im Laboratorium ist ein eindrucksvolles Beispiel für die biogeographische Sonderstellung Helgolands. Die hydrographischen Bedingungen dieses Areals genügen den sporophytischen Phasen heteromorpher Rotalgen, während die jeweiligen Gametophyten wärmere Meere beanspruchen. Am besten bekannt ist das zu Beginn des Jahrhunderts aus Ostasien eingewanderte Heteromorphenpaar TrailliellaBonnemaisonia. Trailliella ist im Sublitoral Helgolands weit verbreitet; Bonnemaisonia

Abb. 12. Porphyropsis imperfecta. Thallusentwicklung bis zum Alter von 10 Tagen, zuletzt in verschiedenem Niveau. B-D Epiphytische und auf dem Schalenboden derselben Kultur gewachsene Thalli, etwa 14 Tage alt. Maßstrecken: $A=50 \mu m_{i} B=200 \mu m_{i} C, D=100 \mu \mathrm{m}$ 

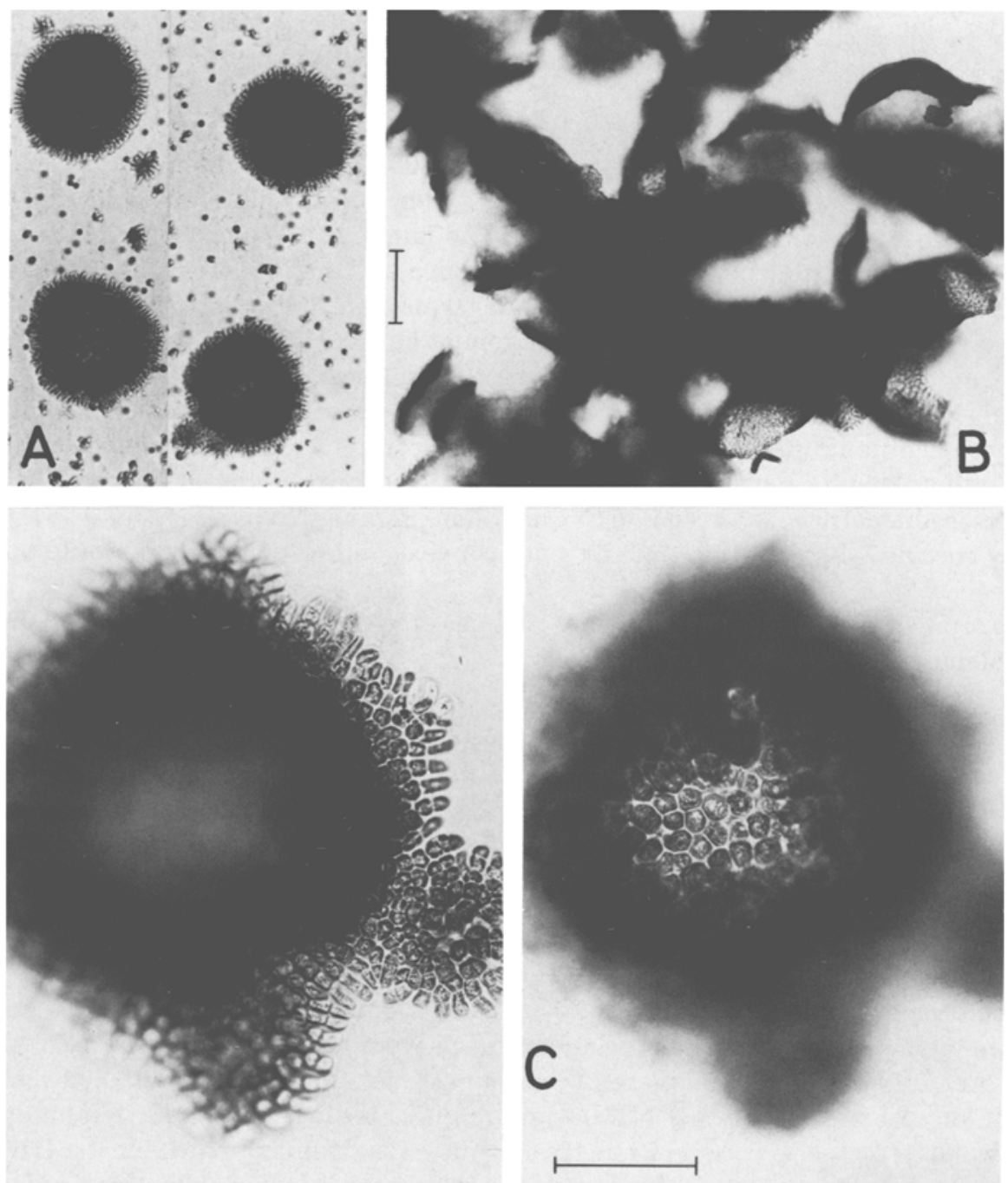

Abb. 13. A, B Porphyropsis imperfecta und Porphyropsis coccinea, in derselben Kulturschale gewachsen, 18 Tage alt. C Porphyropsis imperfecta, vom Boden einer Schale abgelöster und von unten in verschiedener Bildhöhe aufgenommener Thallus. Maßstrecken: A, B $=100 \mu \mathrm{m} ; \mathrm{C}=50 \mu \mathrm{m}$

hamifera trat nur während der sechziger Jahre in unterschiedlicher Häufigkeit auf (Kornmann \& Sahling, 1977). Helminthocladia und Scinaia kommen als kalkbohrende Mikrothalli im Sublitoral vor (Kornmann \& Sahling, 1980); beide Algen waren um die Jahrhundertwende bei Helgoland häufig, sind aber seit mehr als 50 Jahren nicht mehr gefunden worden. Diese Beispiele lassen auf Veränderungen der hydrographischen Verhältnisse schließen, die zum Verschwinden der jeweiligen Gametophyten führten. 


\section{LITERATUR}

Batters, E. A. L., 1900. New or critical British marine algae. - J. Bot., Lond. 38, 369-379.

Berthold, G., 1882. Die Bangiaceen des Golfes von Neapel und der angrenzenden MeeresAbschnitte. - Fauna Flora Golf. Neapel 8, 1-28.

Boney, A. D., 1964. Ecological observations on Erythrotrichia welwitchii (Rupr.) Batt. - Br. phycol. Bull, 2, 327-331.

Børgesen, F., 1915. The marine algae of the Danish West Indies. Rhodophyceae I. - Dansk bot. Ark. 3, 1-80,

Dangeard, P., 1932. Sur quelques Erythrotrichia et Erythrocladia de Banyuls et du Croisic. Botaniste 24, 143-155.

Dangeard, P., 1965. Sur cinq espèces d' "Ulvella". - Botaniste 48, 45-64.

Dangeard, P., 1968. Recherches sur quelques Bangiophycées (Protofloridées). - Botaniste 51, 5-57.

Dixon, P. S., 1973. Biology of the Rhodophyta, Oliver \& Boyd, Edingburgh, $285 \mathrm{pp}$.

Dixon, P. S. \& Murray, S. N., 1981. Life histories in the Erythropeltidaceae (Rhodophyta, Bangiophyceae). - Int. Seaweed Symp. 8, 93-97.

Dixon, P. S. \& West, J. H., 1967. In situ spore germination in Erythrotrichia carnea. - Br. phycol. Bull. $3,253-255$.

Dizerbo, A. H., 1969. Les limites géographiques de quelques algues marines du Massif Armoricain. - Int. Seaweed Symp. 6, 141-149.

Feldmann, J., 1929. Note sur quelques algues marines de Banyuls. - Bull. Soc. bot. Fr. 76, 785-793.

Feldmann, J., 1939. Les algues marines de la côte des Albères. IV. Rhodophycées. - Revue algol. 11, $247-330$.

Feldmann, J., 1954. Inventaire de la flore marine de Roscoff. - Trav. Stn biol. Roscoff (Suppl.) 6, $1-152$.

Fritsch, F. E., 1945. The structure and reproduction of the algae. Univ. Press, Cambridge, 2, 1-939.

Garbary, D. J., Hansen, G. I. \& Scagel, R. F., 1980. A revised classification of the Bangiophyceae (Rhodophyta), - Nova Hedwigia 33, 145-166.

Hamel, G., 1924. Floridées de France - Bangiales. - Revue algol. 1, 278-292; 427-457.

Heerebout, G. R., 1968. Studies on the Erythropeltidaceae (Rhodophyceae - Bangiophycidae). Blumea 16, 139-157.

Kapraun, D. F., 1980. An illustrated guide to the benthic marine algae of Coastal North Carolina. I. Rhodophyta. UNC Press, Chapel Hill, 206 pp.

Kornmann, P., 1984. Erythrotrichopeltis, eine neue Gattung der Erythropeltidaceae (Bangiophyceae, Rhodophyta). - Helgoländer Meeresunters. 38, 207-224.

Kornmann, P. \& Sahling, P.-H., 1977. Meeresalgen von Helgoland. Benthische Grün-, Braun- und Rotalgen. - Helgoländer wiss. Meeresunters. 29, 1-289.

Kornmann, P. \& Sahling, P.-H., 1980. Kalkbohrende Mikrothalli bei Helminthocladia und Scinaia (Nemaliales, Rhodophyta). - Helgoländer Meeresunters. 34, 31-40.

Kornmann, P. \& Sahling, P.-H., 1983. Meeresalgen von Helgoland: Ergänzung. - Helgoländer Meeresunters. $36,1-65$.

Kylin, H., 1925. The marine red algae in the vicinity of the Biological Station at Friday Harbor, Wash, - Lunds Univ. Årsskr. (N. F., Avd. 2) 21 (9), 1-87.

Kylin, H, 1956. Die Gattungen der Rhodophyceen. Gleerup, Lund, $673 \mathrm{pp}$.

Levring, T., 1937. Zur Kenntnis der Algenflora der Norwegischen Westküste. - Lunds Univ. Årsskr. (N. F, Avd. 2) 33 (8), 1-147.

Murray, S. N., Dixon, P. S. \& Scott, J. L., 1972. The life history of Porphyropsis coccinea var, dawsonii in culture. - Br. phycol. J. 7, 323-333.

Newton, L., 1931. A handbook of the British seaweeds. British Museum, London, $478 \mathrm{pp}$.

Nichols, H. W. \& Lissant, E. K. 1967. Developmental studies of Erythrocladia Rosenvinge in culture. - J. Phycol. 3, 6-18.

Parke, M., 1953. A preliminary check-list of British marine algae. - J. mar. biol. Ass. U. K. 32, 497-520.

Rosenvinge, L. K., 1909, 1917, 1923-24, 1931. The marine algae of Denmark. Contributions to their natural history. Vol. 1, Rhodophyceae. P. 1-4. - K. danske Vidensk. Selsk. Skr., 7 (Række 7), $1-630$. 
Schmitz, F, 1896. Bangiaceae. In: Die natürlichen Pflanzenfamilien. Hrsg. von A. Engler \& K. Prantl. Engelmann, Leipzig, 1 (2), 307-316.

Smith, G. M.r 1944. Marine algae of the Monterey Peninsula. Stanford Univ. Press, Stanford, 622 pp. Sundene, O., 1953. The algal vegetation of Oslofjord. - Skr. norske Vidensk Akad. (Mat.-naturv. Kl.) 1953 (2), 1-244.

Womersley, H. B. S. \& Conway, E., 1975. Porphyra and Porphyropsis (Rhodophyta) in southern Australia. - Trans. R. Soc. S. Aust. 99, 59-70. 